\section{Determination of Crystal Lattice Constants by Electron Diffraction}

THe accuracy of the determination of crystal lattice constants by electron diffraction has hitherto been limited by virious sources of error, chief of which is that due to the lack of precision attendant upon the measurement of high voltages. We have recently succeeded in eliminating this and other sources of error in the following manner :

A double specimen, mounted as required either for transmission or reflection, is employed. One half consists of a substance the lattice constants of which are known, having been determined by exact $\mathrm{X}$-ray analysis; the other half of the specimen consists of the substance of unknown lattice constants. The shutter of the diffraction camera is in two halves each capable of indspendent operation. The electron

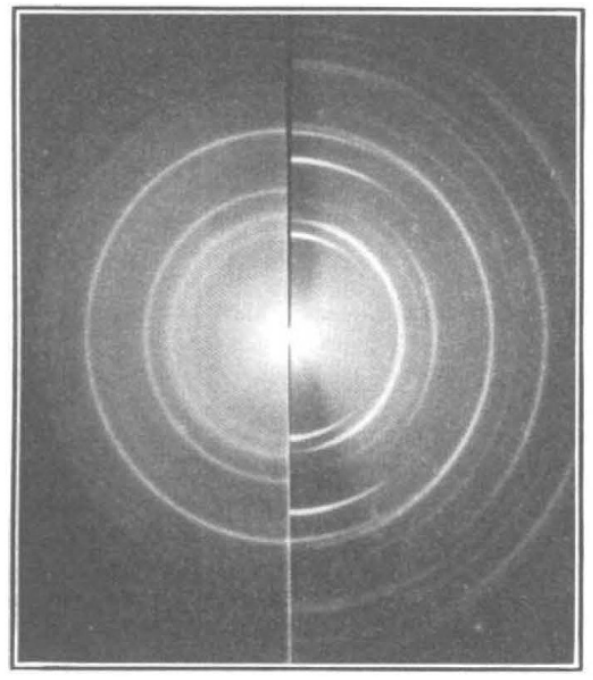

HIG. 1.

beam penetrates or impinges upon one half of the specimin and the corresponding electron diffraction pattem is recorded upon one half of the photographic plate. Thereupon the specimen is either traversed through the beam or rotated in the azimuthal plane, thus bringing the second half of the specimen under examination. The hitherto unexposed half of the photographic plate is now exposed. The accompanying reproduction (Fig. 1) of a transmission photograph obtained in the manner outlined above will serve to make clear the principle underlying the method, which is analogous to the use of the iron-arc reference in spectroscopy.

A more detailed account of this method will be published shortly.

(i. I. FINCH.

A. G. QUarRELL.

Imperial College of Science and Technology, South Kensington, S.W.7. May 11.

\section{Selective Lattice Distortion in Wires under Torsion}

$\mathrm{X}$-RAY analysis of metals affords a moans of detecting the type of internal strain which is accompanied by distortion of the crystal lattice. The distortion is reflected in the X-ray diffraction photographs in that it causes a broadening of the spectrum lines; the effect has been noted for metals which have been cold-worked or which contain a highly dispersed solute.

I find that lattice distortion is produced also in wire (copper, nickel) under torsion, and a point of particular interest appears. Since torsional stress can be applied gradually, the rate of broadening of lines from different planes can be compared. It is found first, that the (420) planes distort more rapidly than do the (33I); and, secondly, the lines do not broaden continuously with increasing stress, but exhibit instead alternating periods in which their definition is partially recovered.

The selective distortion of the planes is of such an order that the (331) $K \alpha_{1} \alpha_{2}$ doublet is easily discernible after the (420) doublet has diffused into a single broad line. The recovery effect is most marked on the (331) line; the doublet diffuses and reappears, though to a less and less degree, as the torsion of the wire is increased.

The observations implement the view that lattice distortion grows, as stress is applied, to the maximum characteristic of the metal; that the more highly distorted grains disrupt, and then tend to re-crystallise, presumably adopting a more convenient orientation, in a temporarily less distorted state. The latter stage would coincide with the recovered definition of particular lines.

\section{The National Physical Laboratory, Teddington, Middlesex. May 8.}

\section{Extension of the Visible Absorption System of $\mathrm{NO}_{2}$ to Longer Wave-Lengths}

IN two recent letters to NAture, Cassie and Bailey ${ }^{1}$ as well as Harris, Benedict and King ${ }^{2}$ report new investigations of the infra-red and ultra-violet absorption spectrum of $\mathrm{NO}_{2}$. We should like to remark that we have recently made new investigations of the visible absorption system of $\mathrm{NO}_{2}$ which, as is well known, is extremely complicated. In consequence of this, neither its vibrational nor rotational structure has as yet been analysed. A possible explanation for this complicated structure is that, in consequence of the Franck-Condon principle, chiefly the higher vibrational levels in the upper state are excited.

On this assumption it was to be expected that the system would extend to longer wave-lengths with sufficient absorbing length and there become more simple. This proved in fact to bo the case. We have found a rather simple series of bands starting at about $8900 \mathrm{~A}$. and extending to shorter wavelengths. The distance between tho main bands is about $740-730 \mathrm{~cm}^{-1}$. This must be a vibrational frequency of the upper state.

We are engaged now in the investigation of these new bands with longer absorbing layers and with high resolution. On account of the absence of overlapping of different bands in the region now being investigated, it seems very likely that it will be possible to analyse the vibrational and rotational structure.

Physikalisches Institut

JAMES CURRY.

G. Herzberg.

der Technischen Hochschule,

Darmstadt, Germany. May 6.

1 YATURE, 131, 239, Feb 18, 1933.

' YATTRE, 131, 621. April 29, 1933. 